\title{
Optimization of the microstrip gas counter
}

\author{
Presented by F.G. Hartjes \\ M.H.J. Geijsberts, F.G. Hartjes, J.G. Pannekoek, J. Schmitz and F. Udo \\ NIKHEF-H, Amsterdam, The Netherlands
}

\begin{abstract}
A study has been made to optimize the characteristics of the microstrip gas counter for use as a tracker at the LHC. In this framework we found that two of the most important design parameters of the detector are given by the choice of the couriter gas and the width of the gas gap. In this report we deduce, using measurements done with a UV laser and a particle beam, two most suited mixtures which consist of xenon, di-methyl-ether and $\mathrm{CO}_{2}$. We also derive the optimum width of the gas gap. With these results an efficient event tagging will be possible at the LHC bunch frequency.
\end{abstract}

\section{Introduction}

In 1987 Oed [1] introduced the microstrip gas counter (MSGC) as an ionization detector. Hereafter, Angelini et al. [2] showed its capability of detecting minimum ionizing particles, giving way to application of the MSGC as a tracker in high energy physics. In another paper [3] they showed that because of the high rate capability (a few hundreds of $\mathrm{kHz}$ per $\mathrm{mm}^{2}$ ), such a tracker should be capable of operating in an environment with a very high particle flux, like the vicinity of the interaction point at the new generation of high luminosity colliders SSC and LHC.

Basically, a MSGC consists of an insulating substrate on which thin conductive strips, alternatively anode and cathode, are deposited (fig. 1). The anode strips are typically 5-10 $\mu \mathrm{m}$ wide with a pitch of 200 $\mu \mathrm{m}$. Free electrons, liberated by a traversing particle in the gap between the substrate and a drift electrode, drift towards the substrate where, in the vicinity of the anode strips, a gas avalanche occurs. The position

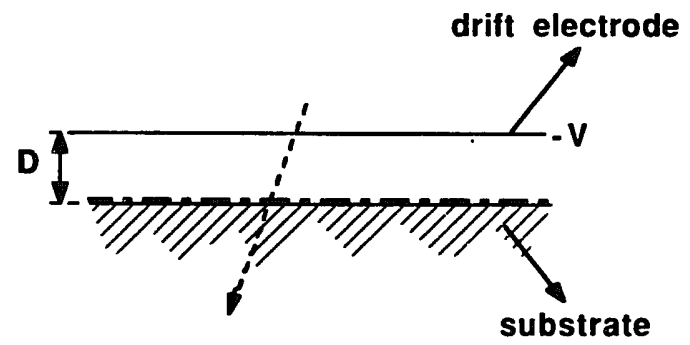

Fig. 1. Schematic cross section of a microstrip gas counter. The drift electrode with potential $-V$ creates a drift field across the gas gap with width $D$. accuracy for minimum ionizing particles amounts to about $30 \mu \mathrm{m}$.

In 1988 a program has been started at NIKHEF to develop MSGCs for use in a tracker at the LHC [4]. For the investigations presented in this report, we concentrated on three very important properties for an LHC detector: 1) short charge collection time, preferably such that each detected event can be unambiguously linked to a specific bunch crossing, 2) capability of operation in a high magnetic field (up to $4 \mathrm{~T}$ ) and 3) good accuracy and double track separation. In the next section we will show that these properties depend mainly on the kind of the used counter gas.

Hereafter, we show the outcome of a search for the best counter gas and the results from a test at a particle beam. We will also deduce the optimal value for the width of the gas gap for application as a tracker at the LHC.

\section{Optimization of the MSGC counter gas in an LHC tracker}

The three properties of the MSGC, mentioned in the previous section, which have to be optimized, determine the choice of the used counter gas. We assume here that the operation is done under atmospheric pressure.

1) Short charge collection time.

To optimize this property, we have to search both for the highest drift velocity and for the most narrow gas gap. The latter we can only apply using a gas with the highest primary ionization in combination with sufficiently high gas gain, to maintain a proper efficiency. Note that here the efficiency is only determined by the 
number of primary clusters in the gas, under the assumption that the gas gain is a few times higher than the input noise of the preamps expressed in number of electrons.

2) Operation in a high magnetic field.

Only at a small Lorentz angle the effect of the magnetic field on the detector performance can be neglected. Therefore, when operating at high magnetic fields such as $4 \mathrm{~T}$ while $E$ and $B$ are not parallel, the mobility $\mu=w / E$ has to be as low as possible, where $w$ is the drift velocity, $B$ the magnetic field and $E$ the drift field.

3) Accuracy and double track separation.

Assuming that in general for a MSGC the resolution is mainly determined by the width and the content of the electron cloud, we will get the best accuracy for a gas with the highest total number of primary electrons and the lowest transverse diffusion. It is obvious, that for the best double track separation only a small diffusion is relevant. Note however, that this consideration is only vaiid as long as for most events the electron cloud is distributed over more than one anode strip, i.e. when the dimension of the electron cloud exceeds the pitch of the anode strips.

Summarizing we can state that for the best operation as a tracker at the LHC, we have to optimize the following five parameters of the counter gas:

1. The drift velocity as high as possible.

2. The primary ionization, both in numbers of clusters and in total number of electrons, to the highest level.

3. The gas gain a few times higher than the number of electrons of equivalent input noise of the preamps.

4. The mobility on a very low level. (In combination with 1. this implies that we can only operate at a high drift field.)

5. The transverse diffusion as low as possible.

An additional condition has to be set on the granularity of the strip pattern to avoid degradation of the resolution and the double track separation:

6. The anode pitch has to be smaller than the width of the electron cloud.

\section{Results from tests using a UV laser and a radioac- tive source}

In an early stage we skipped a lot of the commonly used counter gases like argon, methane and ethane which would obviously not lead to our goal. Instead, we confined ourselves to mixtures containing the densely ionizing gases xenon and di-methyl-ether (DME), which already have been used successfully for a MSGC [3], and $\mathrm{CF}_{4}$ and $\mathrm{CO}_{2}$.

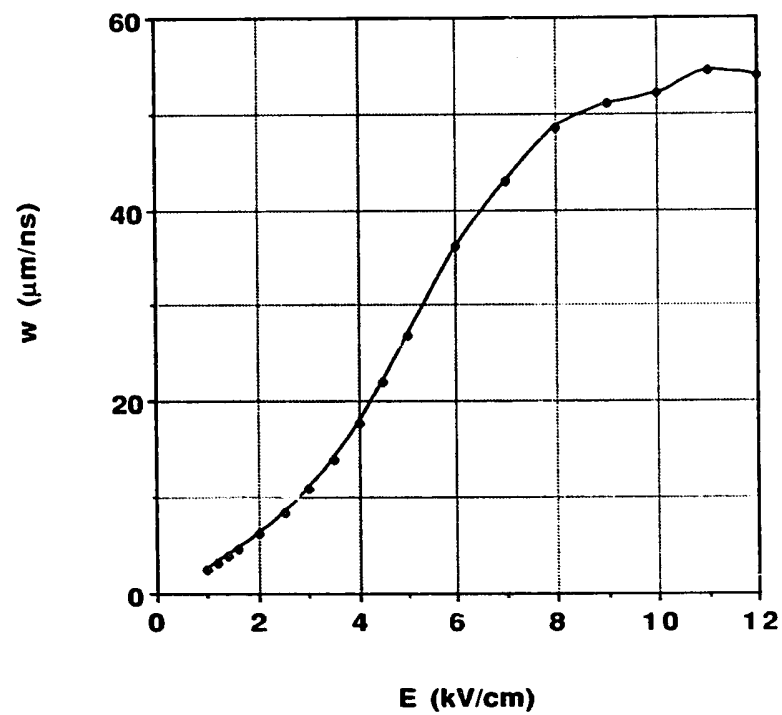

Fig. 2. Measured drift velocity $w$ as a function of the electrical field $E$ for DME.

Using a UV nitrogen laser *, we measured the drift velocity of an extended group of mixtures of these four gases. For most of these mixtures, we also made a qualitative comparison of the maximum gas gain that could be obtained before a HV break down sets in.

The results will be extensively described in ref. [5]. In this report, we limit ourselves to the general behaviour for each of the four gases which we extracted from the drift velocity and gas gain measurements and from literature.

Xenon is a gas with excellent primary ionization [6], but, at high electrical fields, the drift velocity falls down to less than $50 \mu \mathrm{m} / \mathrm{ns}$.

DME has a high density of primary ionization clusters $(55 / \mathrm{cm})$ [7], a moderate total ionization density of $150 \mathrm{e}^{-} / \mathrm{cm}$, and gives the highest gain of all examined organic quenchers. Its mobility is very low (fig. 2). However, for pure DME the drift velocity even at high fields does not exceed $\approx 55 \mu \mathrm{m} / \mathrm{ns}$ at which level it tends to form a plateau.

$\mathrm{CF}_{4}$ has a high drift velocity, but only at low electrical fields. Moreover, at fields above $6 \mathrm{kV} / \mathrm{cm}$, a strong absorption of free electrons sets in. Therefore, we exclude this gas for use in a MSGC.

$\mathrm{CO}_{2}$ has a low and more or less constant mobility over a wide range of electrical fields, so high drift velocities can be reached while the Lorentz angle is low. However, its total ionization density is limited to $\approx 90 \mathrm{e}^{-} / \mathrm{cm}[6]$, and the maximum attainable gas gain was moderately low.

By combining the general properties of some of these gases, we finally found two mixtures which are

\footnotetext{
* NIKHEF type IA.
} 


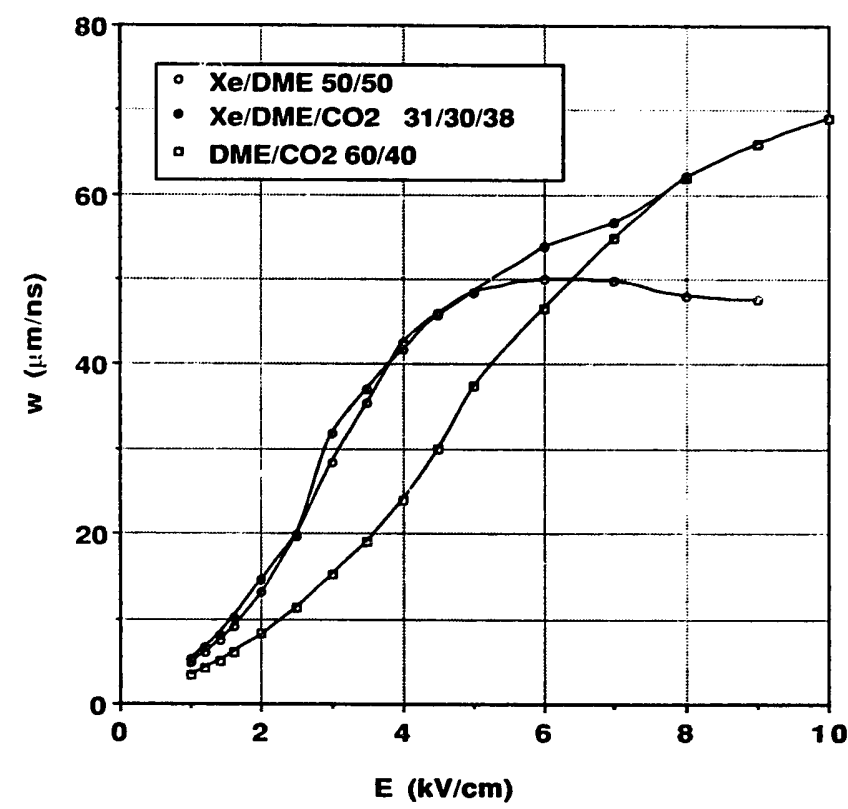

Fig. 3. Measured drift velocity $w$ as a function of the electrical field $E$ for mixtures of xenon, DME and $\mathrm{CO}_{2}$.

the best suited for high rate tracking. DME, with eventually an admixture of xenon, has the best characteristics from the point of view of primary ionization cluster density, ultimate gas gain, mobility and diffusion. Its moderate drift velocity can be extensively raised by adding $\mathrm{CO}_{2}$, as shown in fig. 3. At a $\mathrm{CO}_{2}$ concentration $\geq 30 \%$, the drift velocity curve of a DME/xenon mixture does not show any longer a plateau, but continues to rise for high electrical fields. Therefore, we end up with two most suited mixtures: $\mathrm{DME} / \mathrm{CO}_{2} 60 / 40$ and $\mathrm{Xe} / \mathrm{DME} / \mathrm{CO}_{2} 30 / 30 / 40$.

In the latter mixture, the replacement of half of the DME by xenon gives the advantage of a lower inflammability. The increased level of diffusion from xenon may improve the resolution in cases where, due to the small dimensions of the electron cloud, the anode pitch becomes the limiting factor.

\section{Resulis from a particle beam test}

The resolution and efficiency for DME $/ \mathrm{CO}_{2} 60 / 40$ was measured in an $8 \mathrm{GeV} / c$ pion beam at CERN. The detector had a gas gap of $2.8 \mathrm{~mm}$ wide and an anode pitch of $200 \mu \mathrm{m}$. The angle of incidence was parallel to the normal. Fig. 4 and 5 show that the resolution for minimum ionizing particles is around $\mathbf{3 0}$ $\mu \mathrm{m}$ while the efficiency, depending on the applied software threshold, exceeds $95 \%$. At a cathode strip voltage of $-720 \mathrm{~V}$ we measured as an average signal $1.8 \times 10^{5}$ electrons from the anode strips. From this we

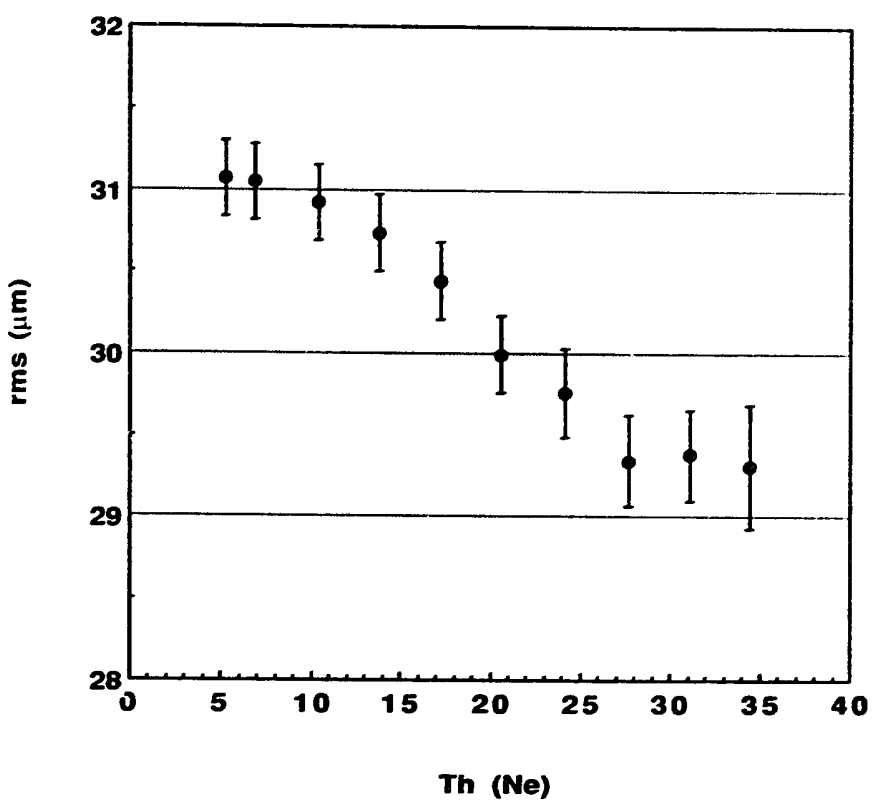

Fig. 4. Measured resolution rms as a function of the threshold Th expressed in number of primary electrons $N_{c}$ for DME/ $\mathrm{CO}_{2} 60 / 40$.

get, under the assumption of 35 electrons along a 2.8 $\mathrm{mm}$ long track, a gas gain of about 5000 . Note that the applied cathode voltage of $-720 \mathrm{~V}$ was considerably above the minimum operating voltage of $-770 \mathrm{~V}$, so the maximum gain of this gas is more than sufficient.

Depending on the threshold, more than $50 \%$ of the events were captured by not more than one anode strip. Obviously, for this gas the dimension of the

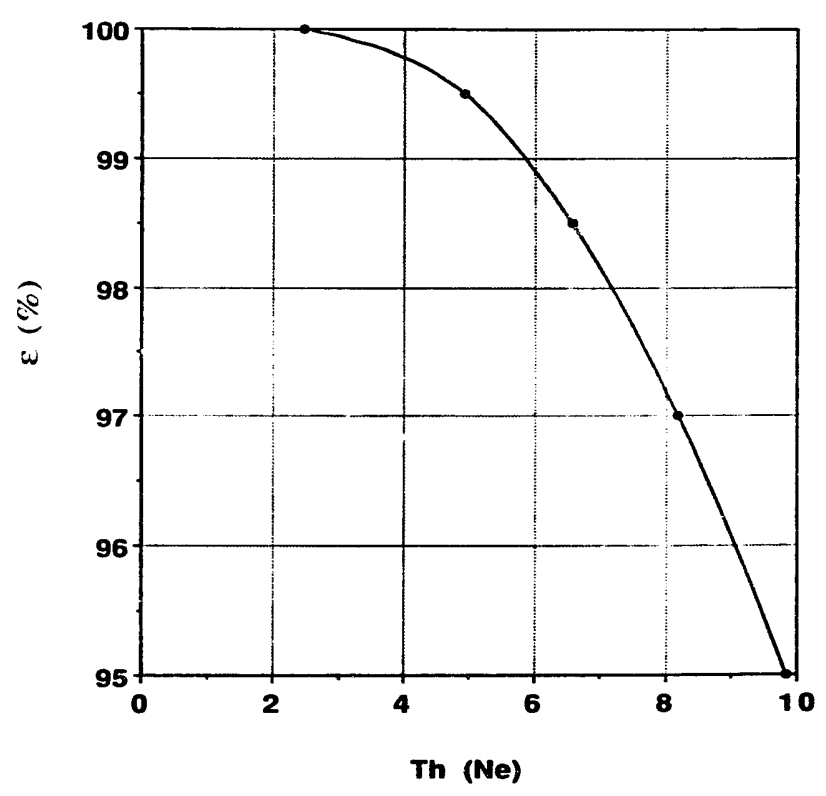

Fig. 5. Measured efficiency $\varepsilon$ as a function of the threshold Th expressed in number of primary electrons $N_{\mathrm{e}}$ for DME/ $\mathrm{CO}_{2} 60 / 40$. 


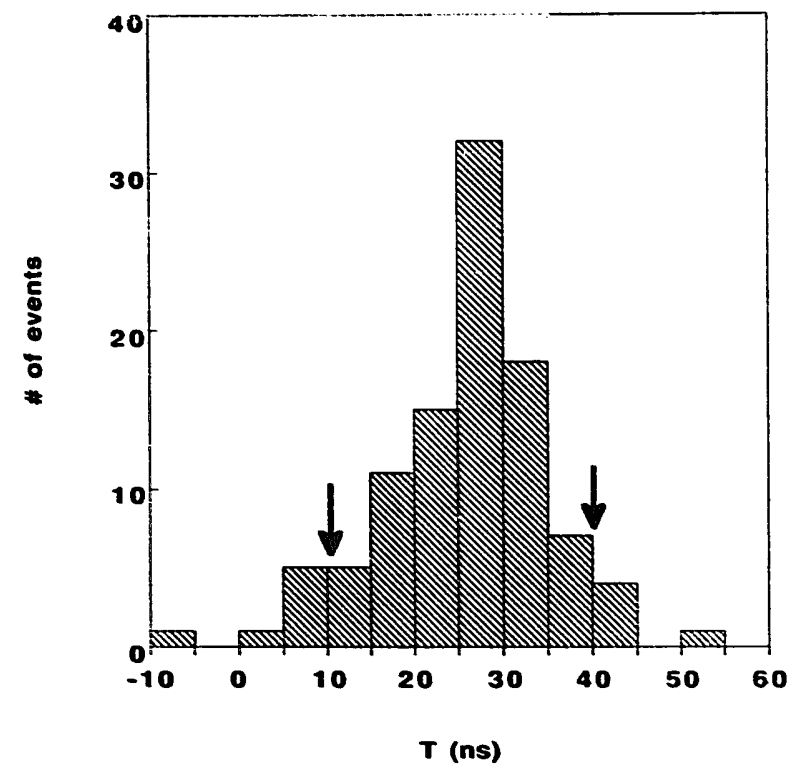

Fig. 6. Histogram of the measured arrival time $T$ for a MSGC with a gas gap of $2.8 \mathrm{~mm}$ for $\mathrm{DME} / \mathrm{CO}_{2} 60 / 40$ at a drift field of $8 \mathrm{kV} / \mathrm{cm}$. An area of $30 \mathrm{~ns}$ wide, as marked by the arrows, contains $88 \%$ of the events.

electron cloud was smaller than the anode pitch, leading to a degradation of the accuracy and the need to correct the measured data for systematic errors. For the presented results this was done using a combined linear-sinusoidal correction function.

To investigate the timing properties, the arrival time of 100 pulses from a single anode strip was measured by a digital oscilloscope. The pulses were digitized using a constant fraction discriminator to avoid any influence from the amplitude. The result in fig. 6 shows that a fraction of $88 \%$ of the events, as indicated by the two arrows, is located within a $\mathbf{3 0}$ ns wide time slot. It is obvious that, when scaling the applied gap width of $2.8 \mathrm{~mm}$ down to $1 \mathrm{~mm}$, more than $90 \%$ of the events can be expected to be confined within a 16 ns time slot which is the bunch crossing period of the LHC. The process of charge collection is thus fast enough to permit a reliable event tagging.

\section{Optimization of the gas gap width}

From the requirement of a short charge collection time, we have learned that the gas gap has to be as narrow as possible. We will now discuss how the other desired detector properties are affected by the width of the gas gap.

The Lorentz angle, in first approximation, can be expected to be independent of the gas gap width. The shift of the measured position due to the magnetic field scales with the gap width, so a narrow gap will be advantageously.

The resolution can be expected to be independent of the gap width, as long as the width of the electron cloud upon arrival at the substrate surface is mainly ruled by diffusion. This behaviour can be understood by taking into account that, while reducing the width of the gas gap, the deterioration of the resolution due to the reducing number of primary electrons, is counterbalanced by the improving resolution from the reduced dimension of the electron cloud. Therefore, with respect to the resolution for perpendicular tracks, the width of the gas gap may have any value. Note that for slanting tracks whose projection is parallel to the strips, better results can be expected due to the improved electron statistics.

For the double track separation, reducing the gas gap will also reduce the dimension of the gas cloud since this parameter is mainly ruled by diffusion. The double track resolution thus improves when reducing the gap width. However, when using a low diffusion gas, the presently used anode pitch of $200 \mu \mathrm{m}$ is already the limiting factor for gas gaps of $3 \mathrm{~mm}$.

The efficiency of course, will deteriorate when reducing the gas gap. For a given width of the gas gap, the theoretical highest efficiency $\varepsilon$ follows from the average number of primary cluster $\boldsymbol{n}$ according to

$\varepsilon=1-\mathrm{e}^{-n}$

Therefore, assuming for each of the two best mixtures an ionization density of 35 clusters per $\mathrm{cm}$, for a gap of $1 \mathrm{~mm}$ we would still get an efficiency of $97 \%$ with a sufficiently high gas gain.

Will it be possible to obtain such a gain? For the gas mixture used for the beam test described in the previous section, a gain of at least $10^{4}$ was already possible for a $4.5 \mathrm{~mm}$ wide gas gap, which is at least several times higher than the noise of most integrated preamp/multiplexer circuits. We could reach such a high gain not only because of a proper choice of the gas, but also because of the high value of the applied drift field $(8 \mathrm{kV} / \mathrm{cm})$. The mechanism of this behaviour can be understood from the following.

Because the drift field induces a positive charge over all conductors on the substrate, a higher drift field will lead to a higher positive charge on the anodes. As a consequence, the gas gain, which is mainly ruled by the anodic charge, will rise. In contrast to this, the increased drift field will reduce the charge density on the cathodes, resulting in a higher breakdown voltage and thus in a higher maximum gas gain.

Tests done in the laboratory with a decreasing drift field confirm this assumption: while bringing the field down and leaving the cathode voltage on the same high 
level, the gas gain drops and often a HV break down occurs.

When reducing the gap width, especially for narrow gaps another positive effect on the gas gain may also play a role. From the Paschen curves [8], which give the relation between the breakdown voltage $U$ and the electrode distance $D$, we can see that, for $D$ in the $\mathrm{mm}$ range, in the first approximation the breakdown voltage is given by

$l=\alpha D+200 \quad(D \geq 10 \mu \mathrm{m})$,

where $U$ is expressed in volts and $\alpha$ is a constant depending on the gas. For the breakdown field $E$ we get thus

$E=\alpha+200 / D$,

where $E$ is expressed in volts per unit of length.

Therefore, when reducing the gap width for instance from $4.5 \mathrm{~mm}$ to $1 \mathrm{~mm}$, the drift field can be increased by more than $1.5 \mathrm{kV} / \mathrm{cm}$ which will lead again to a higher gain.

Concluding we can thus state, that, for application as an LHC tracker, timing and double track separation do both improve when reducing the gas gap. Resolution is expected to remain unaffected. Efficiency gives the only lower limit of about $1 \mathrm{~mm}$ on the gap width which originates from the primary cluster statistics.

\section{Conclusion}

We have demonstrated that the performance of MSGCs in an LHC tracker largely depends upon the choice of the counter gas. The most suited mixtures consist of $\mathrm{DME} / \mathrm{CO}_{2}$ and $\mathrm{Xe} / \mathrm{DME} / \mathrm{CO}_{2}$. When a high drift field is chosen in combination with a narrow gas gap, a reliable event tagging can be expected while the resolution still remains at a high level.

\section{References}

[1] A. Oed, Nucl. Instr. and Meth. A263 (1988) 351.

[2] F. Angelini et al., Nucl. Instr. and Meth. A283 (1989) 755.

[3] F. Angelini et al., Proc. ECFA study week in Barcelona, eds. E. Fernandez and G. Jar)skog, CERN 89-10 (1989) p. 465.

[4] F. Hartjes, B. Hendrikser. and F. Udo, Nucl. Instr. and Meth. A289 (1990) 384.

[5] M. Geijsberts, F.G. Hartjes, J.G. Pannekoek, J. Schmitz and F. Udo, Tests of the performance of microstrip gas counters with different gas mixtures internal report H/KHEF-H/91-23.

[6] F. Sauli, Internal report CERN 77-09, 3 May 1977.

[7] R. Bouclier et al., Nucl. Instr. and Meth. A283 (1989) 509.

[8] A. Roth, Hochspannungstechnik (springer, Wien, 1965) fig. 50/1, p. 259. 